\title{
Non-Hodgkin's lymphoma presenting as 'chronic active toxoplasmosis'
}

\author{
N.J. Dudley ${ }^{1}$ and A.H. Balfour ${ }^{2}$ \\ ${ }^{1}$ Infectious Diseases Unit, Seacroft Hospital, Leeds and ${ }^{2}$ The Toxoplasmosis Unit, Regional Public Health \\ Laboratory, Leeds, UK.
}

\begin{abstract}
Summary: We report the case of a 58 year old man in whom an unusually prolonged infection with Toxoplasma gondii was the presenting feature of an underlying non-Hodgkin's lymphoma. This case demonstrates the particular usefulness of the IgM ELISA test in monitoring disease activity.
\end{abstract}

\section{Introduction}

Toxoplasmosis is a recognized complication of lymphoma in both adults and children. ${ }^{1-3}$ It may arise de novo or secondary to reactivation of latent infection and has a tendency to infect the central nervous system. ${ }^{4-7}$ Infection is more commonly associated with Hodgkin's disease than nonHodgkin's lymphoma. ${ }^{1,3}$ The explanation for this may lie with the type of impaired immunity classically associated with each of these conditions, and the fact that immunity to toxoplasma depends principally on T-lymphocytes and to a much lesser degree on antibody production. ${ }^{8}$ In Hodgkin's disease there is classically a defect in cell-mediated immunity. ${ }^{9-12}$ Humoral immunity remains intact until advanced stages of disease have been reached, ${ }^{13}$ although it has been shown that antibody production is impaired in splenectomized patients following radiotherapy or chemotherapy. ${ }^{14,15}$ In contrast to this, non-Hodgkin's lymphoma is classically associated with a defect in humoral immunity, although in advanced stages cellular immunity may also be impaired. ${ }^{9,16}$

We report on a patient in whom a prolonged infection with Toxoplasma gondii was the presenting feature of the lymphoma. This case illustrates how continuing disease activity may be missed by relying on toxoplasma kit tests used in non-specialized laboratories. It underlines the importance of sending sera for more specialized tests, especially when titres remain elevated and the illness does not follow the anticipated clinical course.

Correspondence: N.J. Dudley, M.B., Ch.B., M.R.C.P., City Hospital, Hucknall Road, Nottingham NG5 1PB, UK.

Accepted: 30 June 1988

\section{Case report}

A 58 year old man was referred to the infectious diseases unit with a 22 month history of generalized lymphadenopathy, recurrent abdominal pain, lethargy and a toxoplasma latex agglutination (Eiken Toxoreagent) (LA) titre of 1,024 . The lymphadenopathy had developed over a few weeks at which time initial investigations showed an absolute lymphocytosis of $8.7 \times 10^{9} / 1$, some of the lymphocytes being atypical and suggestive of viral infection. Toxoplasmosis was diagnosed by a raised LA titre of 1,024 . Improvement occurred without treatment and the lymphadenopathy became less marked.

Four months later, he was admitted to the local hospital with acute abdominal pain. Pancreatitis was diagnosed for which no precipitating cause was found. Prior to discharge a white cell count showed a lymphocytosis of $6.8 \times 10^{9} / 1$ and a repeat LA titre remained elevated at 2,048. Both this and the original serum were sent for further testing at the Toxoplasma Reference Laboratory, Leeds. Rising titres were reported in the dye test, 16,000 to 64,000 and the IgM enzyme linked immunosorbent assay (ELISA), ${ }^{17} 141$ to 186 EIU (Enzyme Immunoassay Units). These were reported as being consistent with recent infection. During out-patient follow-up he had repeated bouts of abdominal pain thought to be pancreatic in origin and persistent lymphadenopathy. At 12 months the LA titre was still 2,048 and the absolute lymphocytosis remained.

At 22 months his clinical condition had not improved so he was referred for further investigation. Toxoplasma serology showed a dye-test titre of 64,000 and a strongly positive IgM ELISA titre of $108 \mathrm{EIU}$. Once again the absolute lymphocytosis was noted, although this time the blood film was

(C) The Fellowship of Postgraduate Medicine, 1988 
suggestive of early chronic lymphocytic leukaemia or lymphoma. A non-Hodgkin's lymphoma of diffuse pattern of centrocytic-centroblastic cell type was diagnosed from a lymph node biopsy and marrow trephine. No treatment was required for the lymphoma. As elevated IgM levels are normally considered indicative of recent infection, a 3-week course of spiramycin $1 \mathrm{~g}$ b.d. was given. This produced an unusually rapid and dramatic reduction in the degree of lymphadenopathy with associated symptomatic improvement, although on stopping treatment he suffered a relapse which responded to a further 3-month course of spiramycin. The IgM ELISA fell quickly to levels of doubtful significance at 41 EIU after 3 months, although the dye-test titre remained high at 64,000 . The absolute lymphocytosis persisted throughout treatment. Apart from an episode of supraophthalmic zoster, the patient has remained well during a 2-year follow-up period without any recurrence of abdominal pain or lymphadenopathy, and the IgM test has become negative at 15 EIU.

\section{Discussion}

Neither the clinical nor serological progression of the illness were wholly compatible with a diagnosis of acute toxoplasmosis. Lymphadenopathy persisting for almost two years is atypical, the natural history being one of spontaneous resolution of

\section{References}

1. Ruskin, J. \& Remington, J.S., Toxoplasmosis in the compromised host. Ann Intern Med 1976, 84: 193-199.

2. Boguslawska-Jaworska, J., Pisarek, J. \& Chybicka, A. Toxoplasmosis in the course of neoplastic diseases of haematopoietic and lymphatic system in children. Mater Med Pol 1982: 62-67.

3. Vietzke, W.M., Gelderman, A.H., Grimley, P.M. et al. Toxoplasmosis complicating malignancy. Cancer 1968, 21: 816-827.

4. Smith, G.M., Leyland, M.J., Crocker, J. et al. Cerebral toxoplasmosis in a patient with Hodgkin's disease. J Infect 1987, 14: 243-245.

5. Green, J.A., Spruance, S.L. \& Cheson, B.D. Favourable outcome of central nervous system toxoplasmosis occurring in a patient with untreated Hodgkin's disease. Cancer 1980 45: 808-810.

6. Slavick, H.E. \& Lipman, I.J. Brain stem toxoplasmosis complicating Hodgkin's disease. Arch Neurol 1977, 34: 636-637.

7. Hakes, T.B. \& Armstrong, D. Toxoplasmosis. Problems in diagnosis and treatment. Cancer 1983, 52: 1535-1540.

8. Frenkel, J.K. Immunity in toxoplasmosis. Bull Pan Am Health Organ 1985, 19: 354-367. symptoms and lymphadenopathy within a few months. ${ }^{18}$ The diagnosis of the underlying lymphoma explained the presence of the lymphocytosis which had been incorrectly attributed to toxoplasma infection. It also explained why no precipitating cause was identified for the episodes of pancreatitis, this probably being a manifestation of toxoplasma infection as pancreatic involvement is a recognized finding in immunocompromised patients. ${ }^{19}$

The serology was also unusual as a strongly positive IgM ELISA of 108 EIU is certainly abnormal at 22 months, this test normally being negative one year post infection. ${ }^{17}$ Specific antibody of the IgG class can persist for a number of years, ${ }^{20}$ although as this case well illustrates, in the presence of such elevated titres it is important to perform a specific IgM test in order to determine disease activity.

It would appear that the presence of the underlying lymphoma prevented an adequate immune response being mounted against the toxoplasma infection, resulting in a prolonged infection as indicated by persistent lymphadenopathy and elevated IgM titres.

\section{Acknowledgement}

We thank Dr J. Stevenson for allowing us to report on his patient.

9. Hancock, B.W., Bruce, L., Sugden, P. et al. Immune status in untreated patients with lymphoreticular malignancy - a multifactorial study. Clin Oncol 1977, 3: 57-63.

10. Aisenberg, A.C. Manifestations of immunologic unresponsiveness in Hodgkin's disease. Cancer Res 1966, 26: 1152-1160.

11. Young, R.C., Corder, M.P., Haynes, H.A. et al. Delayed hypersensitivity in Hodgkin's disease. $A m J$ Med 1972, 52: 63-72.

12. Hersh, E.M. \& Oppenheim, J.J. Impaired in vitro lymphocyte transformation in Hodgkin's disease. $N$ Engl J Med 1965, 273: 1006-1012.

13. Aisenberg, A.C. \& Leskowitz, S. Antibody formation in Hodgkin's disease. $N$ Engl $J$ Med 1963, 268: 1269-1272.

14. Hancock, B.W., Bruce, L., Ward, A.M. et al. The immediate effects of splenectomy, radiotherapy and cytotoxic chemotherapy on the immune status of patients with malignant lymphoma. Clin Oncol 1977 3: 137-144. 
15. Siber, G.R., Weitzman, S.A., Aisenberg, A.C. et al. Impaired antibody response to pneumococcal vaccine after treatment for Hodgkin's disease. $N$ Engl J Med 1978, 299: 442-448.

16. Miller, D.G., Patterns of immunological deficiency in lymphomas and leukaemias. Ann Intern Med 1962, 57: 703-716.

17. Payne, P.A., Johnson, D.H.M., Balfour, A.H. et al. Public Health Laboratory Service enzyme linked immunosorbent assay for detecting toxoplasma specific igM antibody. J Clin Pathol 1987, 40: 276-281.
18. Krick, J.A. \& Remington, J.S. Toxoplasmosis in the adult. An overview. $N$ Engl $J$ Med 1978, 298: 550-553.

19. Remington, J.S. \& McLeod, R., Toxoplasmosis. In: Braude, A.I. (ed) Medical Microbiology and Infectious Diseases. W.B. Saunders Company, Philadelphia, 1981 , p. 1822.

20. Sabin, A.B. \& Feldman, H.A. Dyes as microchemical indicators of a new immunity phenomenon affecting a protozoan parasite (Toxoplasma). Science 1948, 108: $660-663$. 